\title{
FATORES DETERMINANTES DO CONSUMO DE ALIMENTOS CERTIFICADOS NO BRASIL
}

\author{
Factors to Certified food consumption in Brazil \\ Joice Zagna Valent', Tatiana Regina Vieira², Adriano Bruzza ${ }^{3}$, Renata Gonçalves Rodrigues ${ }^{4}$, Andrea Polidori \\ Celia $^{5}$, Verônica Schmidt ${ }^{6}$ \\ Mestranda em Agronegócios, PPG Agronegócios, Universidade Federal do Rio Grande do Sul (UFRGS), Porto Alegre, RS \\ ${ }^{2}$ Graduanda em Medicina Veterinária, Bolsista Iniciação Científica CNPq-UFRGS \\ 3Mestando em Ciências Veterinárias, PPG Ciências Veterinária - UFRGS \\ ${ }^{4}$ Doutoranda em Agronegócios, PPG Agronegócios - UFRGS \\ ${ }^{5}$ Mestre em Agronegócios, Professora UPF \\ ${ }^{6}$ Doutor, Professora Associada UFRGS
}

\begin{abstract}
Resumo
A diversidade de produtos agroalimentares e os problemas contemporâneos de segurança alimentar conduzem à incerteza sobre a qualidade dos produtos, seja para os diferentes elos da cadeia produtiva, seja para o consumidor final. Neste sentido, o presente estudo objetivou identificar os principais fatores na tomada de decisão dos consumidores de produtos com certificação. Para tanto, realizou-se uma survey com amostragem não probabilística pelo encaminhamento de um questionário on line, que foi respondido por 220 pessoas. Entre os fatores que determinam o consumo de produtos certificados estão: renda mensal, escolaridade e número de pessoas na família. O preço elevado é considerado um dos obstáculos ao consumo de produtos certificados. Verificou-se que existem consumidores que não sabem o que é certificação, o que se constitui em entrave para a decisão de compra dos produtos certificados. Estudos apontam incremento na importância que os consumidores atribuem à certificação após campanhas educativas. Neste sentido, faz-se necessário desenvolver campanhas de informação e de divulgação das características de produtos certificados.
\end{abstract}

Palavras-chave: certificação, alimentos, perfil consumidor

\begin{abstract}
Agri-food products diversity and contemporary problems of food safety are leading to an uncertainty about the quality of products along the production chain. In this regard, the present study aimed to identify main factors in decision making of certified products consumers. To this effect, was performed a non-probabilistic sampling survey by sending an online questionnaire answered by 220 people. Among the factors that determine certified products consumption are monthly income, educational level and number of people in the family. The high price is considered as one of the barriers to certified products consumption. It was found that there are consumers who do not know what certification is. This fact constitutes an obstacle on certified products buying decision. Studies show an increase in importance when consumers attach to certification after educational campaigns. In this sense, it is necessary to develop information and awareness campaigns of the characteristics of certified products.
\end{abstract}

Keywords: certification, foods, consumer profile. 


\section{INTRODUÇÃO}

Certificação e garantia de qualidade são definidos como sistemas de participação voluntária que permite aos stakeholders, envolvidos nas cadeias alimentares, reivindicar que os produtos ou processos cumpram requisitos de qualidade definidos (DRIES e MANCINI, 2006).

Os sistemas de certificação voluntária surgiram como importante mecanismo de governança para garantia da qualidade de alimentos e existem várias razões pelas quais a certificação tornou-se o mecanismo de destaque. Além disso, a certificação pode ser percebida como uma forma de reforçar a confiança do consumidor. Esta confiança tem sido seriamente abalada devido a escândalos alimentares e, dessa forma, os consumidores são assegurados por uma camada adicional de verificação de qualidade (KIMURA, 2010). Isto por que os produtos certificados enquadram-se no conceito de produtos tradicionais de qualidade (VIVAS et al., 2007).

Entre os fatores que influenciam o comportamento do consumidor estão as características sócio demográficas, necessidades, motivações e personalidade, percepções e imagens, os grupos e líderes de opinião, classe social, estilo de vida, cultura e sistemas de valores. Com base nestes fatores, os consumidores avaliam a qualidade dos produtos através de suas percepções e as escolhas têm como base uma escala de valores ponderados. Muitas vezes esta percepção de qualidade dos alimentos se torna complexa e variada, em decorrência das influências culturais, de tradição e, também, como resultado das riquezas e sistemas socioeconômicos. A exemplo da União Europeia, que abrange uma ampla gama de povos, encontra-se uma percepção de qualidade dos alimentos complexa e variada (VIVAS et al., 2007; DUBOIS, 1993; DRIES e MANCINI, 2006).

Entre os principais sistemas de certificação estão a Análise de Perigos e Pontos Críticos de Controle (APPCC) e as séries "ISO", da Internacional Standards Organization, que foram rapidamente adotados pela indústria de alimentos em todo o planeta. Embora o sistema APPCC seja de implantação progressiva e obrigatória nas indústrias de produção de alimentos de origem animal sob inspeção federal no Brasil, os consumidores pouco sabem sobre este sistema. Este arranjo implica em mecanismos internos à garantia de qualidade dos produtos.

Em 1990 foram introduzidas as normas da série ISO 9000, de gestão da qualidade e Garantia de Qualidade, que foram adotadas pela indústria de alimentos em vários países. Em 1996, inseriu-se a série ISO 14000, norma para gestão e certificação ambiental, a qual se aplica às indùstriais, agroindustriais e comércio, entre outros. Em 1992, a União Européia (UE) criou os sistemas de Denominação de Origem Protegida (PDO), Indicação Geográfica Protegida (PGI) e Especialidade Tradicional Garantida (TSG) para promover e proteger alimentos específicos. A produção integrada de frutas (PIF) surgiu da necessidade de se racionalizar o uso de agroquímicos, sem prejuízos à produção e à produtividade das culturas. Os princípios básicos que regem a PIF são: elaboração e desenvolvimento de normas específicas para cada cultura, e caderneta de campo e de pós-colheita, onde devem ser anotadas todas as práticas de manejo adotadas na produção das frutas.

No Brasil, a produção integrada está sendo adotada por todas as fruteiras com expressão comercial no mercado, envolvendo 14 espécies. A maçã foi à primeira fruta a ser manejada de acordo com o sistema PIF, seguida pelas culturas do mamão, manga, uva, caju, melão, maracujá, banana, coco, morango, figo, caqui, goiaba e pêssego, além dos citros, que já possuem instruções normativas, para posteriormente serem certificadas.

Outros movimentos, que procuram a sustentabilidade ambiental e social, voltaram-se para semelhantes esquemas, como os produtos orgânicos e comércio justo (DRIES e MANCINI, 2006; KIMURA, 2010). Entre os processos diferenciados de produção de alimentos, talvez o mais conhecido entre os consumidores seja a produção orgânica.

No Brasil a área ocupada com cultivos orgânicos era de 100 mil hectares em 2003, o que assegurou o segundo lugar na América Latina, após a Argentina que dedicava, então, 3 milhões de hectares aos cultivos orgânicos. O mercado exportador é muito atraente para os produtos orgânicos, especialmente para frutas. Entre as principais frutas exportadas estão: laranja e frutas secas (SP); castanha de caju (NE); Guaraná (AM). Empresas especializadas em produtos orgânicos, como a Organic Farm Foods, têm interesse em identificar os fornecedores no Brasil. Este interesse visa, principalmente, produtores de bananas, mangas, melões e uvas, com a intenção de fomentar o desenvolvimento da atividade mediante o fornecimento de orientação técnica, financiamento a produtores e antecipação do pagamento, além de garantir a comercialização (TIBOLA e FACHINELLO, 2004).

Em países como Austrália, que possui a maior área mundial de produção orgânica (maioria 
pastagens), a indústria de orgânicos é numericamente pequena comparada à indústria convencional (CHANG e ZEPEDA, 2005). Este fator indica que o processo produtivo diferenciado pode aumentar sua representatividade no mercado e, para tanto, faz-se necessário conhecer o potencial e exigências do consumidor.

Grunert (2005) aponta que a pesquisa sobre qualidade e segurança alimentar perpassa por duas correntes de observação em conjunto. Considerando que a primeira corrente lida com o lado da demanda e a segunda trata da oferta correspondente, estas constituem a abordagem econômica tradicional para lidar com questões de qualidade e segurança. Recentemente, uma terceira corrente foi adicionada, esta lida com a questão de como a qualidade e a segurança são percebidas pelos consumidores e como essas percepções influenciam a tomada de decisão de consumo.

Neste sentido, o presente estudo objetivou identificar os principais fatores na tomada de decisão dos consumidores de produtos com certificação.

\section{METODOLOGIA}

Realizou-se um estudo do tipo exploratório e descritivo e, pela extensão de seu campo, trata-se de um levantamento. Segundo a natureza dos dados, é subjetiva, porque coletou opiniões e atitudes dos entrevistados (CERVO e BERVIAN, 1983).

A fonte de coleta de dados foi primária e secundária. Primária, porque teve questões próprias estruturadas para este estudo, e secundária, porque alguns dados apresentados e discutidos tiveram origem na bibliografia científica nacional e internacional.

Para obter os dados de origem primária os autores realizaram uma survey via internet (GIL, 2007). Survey é uma pesquisa de opinião pública que envolve a coleta de dados por meio de entrevistas ou questionários aplicados à uma amostra selecionada da população em estudo, em observância a um protocolo metodológico científico (FREITAS et al., 2000). O questionário foi aplicado à uma amostra do tipo não probabilística por conveniência (THRUSFIELD, 2004). A divulgação da pesquisa foi feita por meio de carta de solicitação para preenchimento do questionário, enviado por e-mail, com o link da pesquisa, de forma aleatória do tipo "bola de neve", consistindo na solicitação de que o grupo inicial de entrevistados reenviasse a seus contatos pessoais de interesse (BARCELLOS et al., 2012), resultando em 220 entrevistas respondidas. Em algumas questões de caráter múltiplo, o questionado podia assinalar mais de uma alternativa.

Os dados de origem secundária foram obtidos por meio de pesquisa bibliográfica. Esta pesquisa busca informações em livros, artigos científicos e outros meios de publicação (RODRIGUES, 2007).

\section{RESULTADOS E DISCUSSÃO}

A diversidade de produtos agroalimentares e os problemas contemporâneos de segurança alimentar conduzem à incerteza sobre a qualidade dos produtos, seja para os diferentes elos da cadeia produtiva, seja para o consumidor final.

Qualidade dos alimentos é um conceito complexo e varia de acordo com a percepção do consumidor. A palavra mais frequentemente associada ao consumo de alimentos é sabor ( $30 \mathrm{a}$ $50 \%)$, entretanto a palavra saúde (20\%) também é mencionada (DRIES e MANCINI, 2006).

Entre os alimentos de origem animal, a carne bovina é uma das mais estudadas quanto à percepção dos consumidores em termos de qualidade. No Brasil, o atributo determinante para compra de carne bovina é a cor, seguida pela maciez e pelo preço. Entretanto, $83 \%$ dos consumidores estariam dispostos a pagar a mais se a carne apresentar algum tipo de certificado e este garantisse informações sobre a qualidade sanitária do produto. No país, a certificação é considerada importante pelos consumidores (cerca de $90 \%$ ) e exigida por mais de $50 \%$ destes, indicando o crescimento do mercado para produtos certificados no Brasil e no mundo (VELHO et al., 2009).

Embora a grande maioria dos brasileiros afirme consumir produtos certificados, uma parcela significativa o faz raramente e nunca (Figura 1). Entretanto, são consenso a necessidade de divulgação dos processos de certificação e suas implicações na qualidade dos produtos, em especial os alimentos de origem animal.

As pesquisas sobre perfil dos consumidores relatam que estes desconhecem os processos de certificação e seus significados. Verificou-se que os consumidores tendem a associar o processo de certificação a um alimento ou produto específico como, por exemplo, rastreabilidade e carne, indicação geográfica de vinhos, certificação ambiental de madeira, entre outros. Tal fato pode ser mais bem percebido quando o consumidor aponta os fatores que o motivam à compra de produtos certificados. 


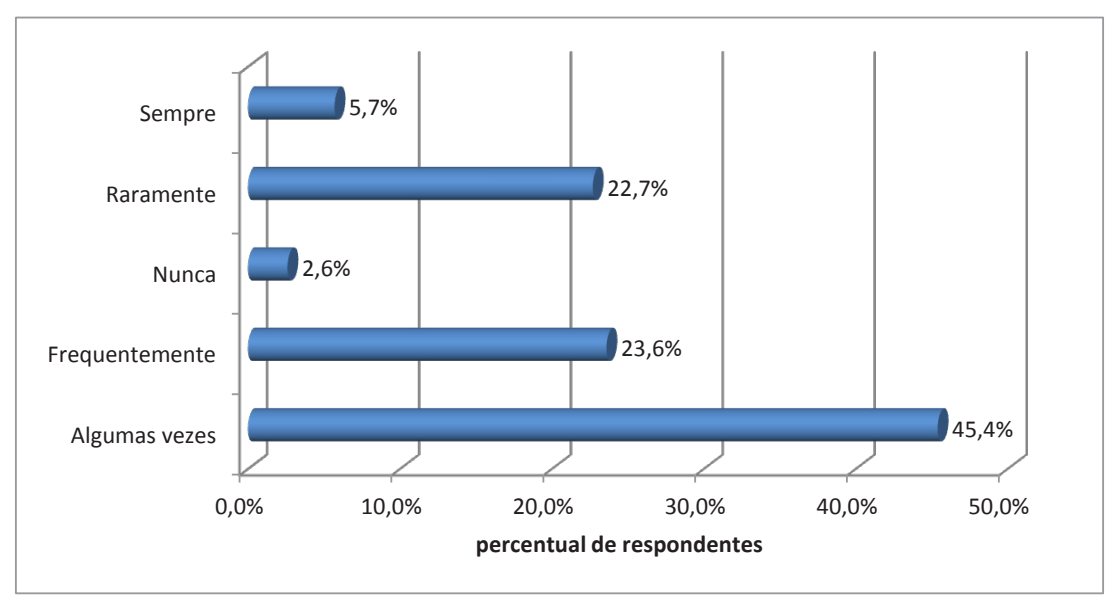

Figura 1: Frequência de consumo de produtos certificados, no Brasil.

Embora os principais fatores de motivação à compra de produtos certificados, para os brasileiros, sejam a segurança alimentar e a credibilidade dos produtos, um pequeno percentual $(2 \%)$ de consumidores ainda não percebe diferença entre os produtos certificados e os demais. Por outro lado, o conceito de certificação ainda não está esclarecido para um percentual maior de consumidores, à medida que estes ainda associam os processos de garantia de qualidade com valor nutritivo, entre outros (Figura2).

Nos últimos anos tem sido observado um interesse crescente do consumidor de carne bovina com garantias de qualidade, tais como designação de origem, rastreabilidade, produção orgânica, certificação de sanidade (THILMANY et al., 2006; VELHO et al., 2009). As denominações de origem protegida (DO), indicações geográficas protegidas (IG) ou as certificações de conformidade (CC) são instrumentos cada vez mais presentes neste mercado de alimentos (SATO, 2009) e os consumidores que conhecem selos de qualidade reconhecem que os produtos certificados possuem atributos diferenciados dos demais (ALCANTARA et al., 2008).

Os processos de certificação mais conhecidos pelos consumidores brasileiros são o orgânico e a denominação de origem (Figura 3).

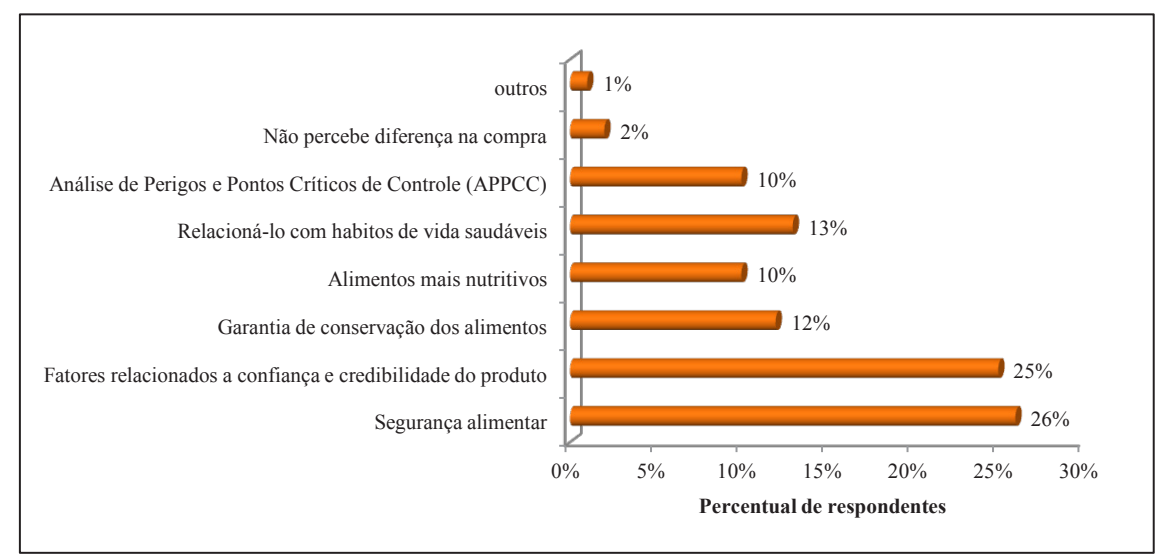

Figura 2: Fatores que motivam os consumidores brasileiros à compra de produtos certificados.

Fonte: autores 


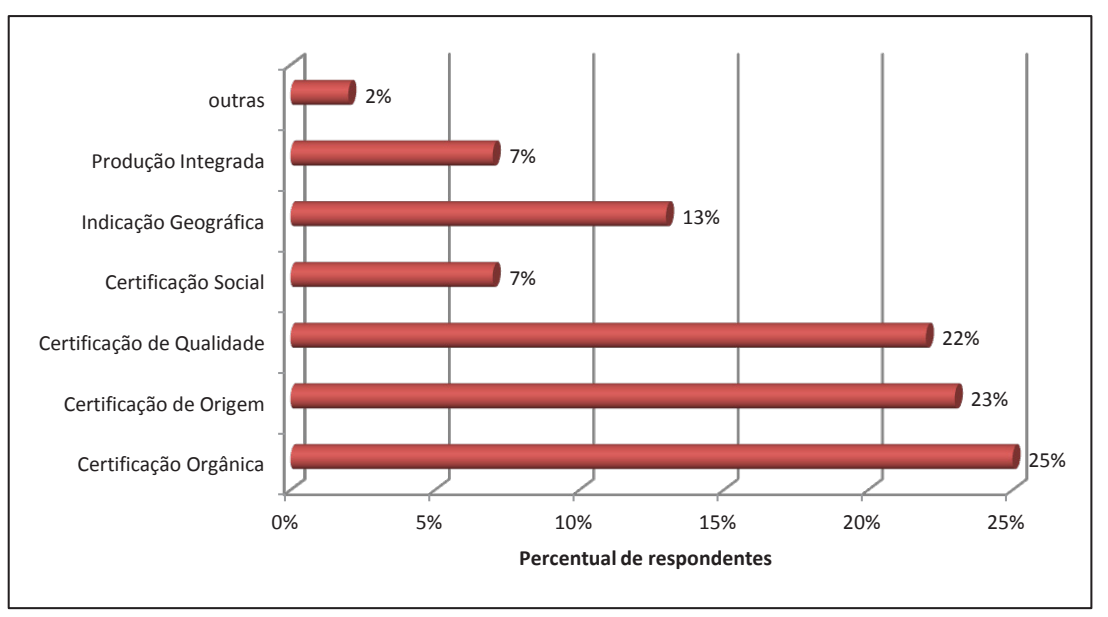

Figura 3: Tipos de certificação conhecida pelos brasileiros.

Fonte: autores

A seguir, são discutidos os tipos de certificação mais referidos pelos respondentes.

\section{PRODUTOS ORGÂNICOS}

É possível perceber que, em geral, os consumidores tendem a confundir produção ecológica, certificação orgânica e certificação ambiental. Entretanto, quando questionados sobre qual o tipo de certificação de alimentos que conhece, os produtos orgânicos encontram-se à frente dos demais alimentos certificados. Talvez por este motivo encontra-se um maior número de pesquisas sobre o perfil de consumidores de produtos com certificação orgânica do que de outros processos. Entre os fatores que se destacam no consumo destes produtos estão os hábitos de vida mais saudáveis, a preocupação com a saúde humana e a segurança alimentar, que está relacionada à ausência de produtos químicos nos alimentos orgânicos. O que chama a atenção sobre o estudo do perfil do consumidor de produtos orgânicos é que este não se alterou com o passar do tempo. Estudos realizados nas décadas de 1980-90 apresentam perfis semelhantes aos observados no presente.

Os consumidores brasilerios de produtos orgânicos são, na maioria, adultos e possuem ensino superior e renda na faixa salarial de 5 a 10 salários mínimos. A maioria dos consumidores gosta do produto e destaca características como sabor, qualidade e aparência. Assim como os consumidores de outros países, associam o consumo à saúde, além do valor nutricional e da ausência de produtos químicos. Em contrapartida, os consumidores apontaram o preço elevado, a pouca diversidade e dificuldade de encontrar os produtos no mercado como fatores limitantes ao consumo. O preço elevado, de acordo com Lombardi et al. (2004), deve-se ao fato do mercado consumidor desses produtos estar em formação, fazendo com que a distribuição seja mais pulverizada do que o normal, acarretando maiores custos de entrega.

O número de brasileiros consumidores regulares de produtos orgânicos é elevado em algumas regiões do país (50\% em Goiânia, 56\% em Minas Gerais, $67 \%$ na Bahia, 75 a $92 \%$ em Santa Catarina). Para aqueles que adquirem estes produtos em supermercados, a garantia sobre a autenticidade dos produtos é observada pela presença de um selo de certificação (KUNESKI et al., 2004), a qual é considerada muito importante pelos consumidores (LOMBARDI et al., 2004; BARROS e FREITAS, 2010). Entretanto, a maioria dos consumidores brasileiros adquirem produtos orgânicos em feiras onde os produtos não possuem, necessariamente, certificação. Nas ferias de produtores, a relação de confiança que se estabelece entre o produtor e o consumidor é o fator preponderante para a escolha dos produtos. Este fato apoia a percepção de diversos pesquisadores de que os consumidores brasileiros confundem ou não sabem diferenciar o produto orgânico dos demais, sendo a referência ao não uso de agrotóxicos ou produtos químicos o marco diferencial.

A garantia de procedência dos produtos, assim como a falta de informação sobre produtos orgâncios, tem sido apontada em pesquisas com consumidores no país. Estes dados reforçam a percepção de que existem dúvidas dos consumidores sobre o processo de certificação.

Do ponto de vista do consumidor, a pro- 
dução orgânica está intimamente relacionada a três áreas fundamentais: meio ambiente, saúde humana e bem-estar animal. Alimentos orgânicos, por meio dos sistemas de certificação, asseguram aos consumidores que não foram utilizados materiais transgênicos ou produtos químicos sintéticos durante o processo de produção e, no caso de produtos de origem animal, que tenham sido respeitados os aspectos de bem-estar dos mesmos (RODRIGUEZ et al., 2002).

Desta forma percebe-se que o consumidor dos produtos orgânicos considera muito mais os aspectos relacionados aos benefícios gerados pelo produto, na sua percepção, do que a valorização pela consciência ambiental e da postura ecológica relacionada a uma certificação como a orgânica. Contudo, o tema demanda mais investigações como, por exemplo, a combinação destes fatores.

\section{CERTIFICAÇÃO POR DENOMINAÇÃO DE ORIGEM}

Para os brasileiros, o consumo de produtos com certificação de origem tem como principais motivações a qualidade dos produtos associados a uma região, apoio à produção local e rastreabilidade (Figura 4).

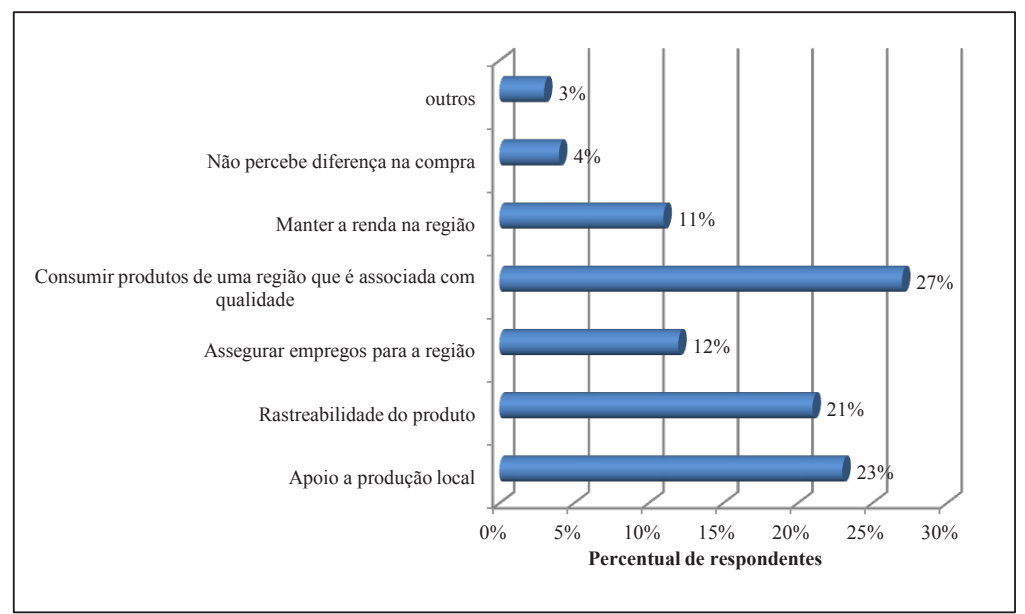

Figura 4: Fatores de motivação à compra de produtos com certificação de origem, no Brasil

Fonte: autores

No Rio Grande do Sul, a busca de produtos de origem conhecida e com garantia de segurança para o consumo, assim como características organolépticas, encontra-se entre os quesitos mais citados na tomada de decisão para compra de frutas. Nesse quesito, particularmente, houve mudança do perfil do consumidor em relação à década de 80 , quando origem e segurança não se apresentavam entre os critérios mais citados (ROMBALDI et al., 2007).

A crescente demanda por produtos de qualidade diferenciada, no Brasil e no mundo, representa uma importante oportunidade para valorização de produtos do meio rural brasileiro com denominações de origem. Experiências de pequenos produtores, como o caso da Queijaria Alpina, evidenciam o potencial de sucesso da pequena produção no Brasil (CAETANO e VALENTE, 2010).

\section{CERTIFICAÇÃO DE SANIDADE ANIMAL}

A certificação de sanidade animal está diretamente relacionada à segurança alimentar do consumidor. A certificação compulsória e a legislação pertinente estabelecem padrões mínimos de qualidade para produtos de origem animal, os quais são específicos para cada país e podem ter por base exigências internacionais e servir, inclusive, como barreira não tarifária às exportações. Os estabelecimentos beneficiadores e manipuladores da matéria prima de origem animal deverão seguir as normativas estabelecidas em nível federal, estadual ou municipal.

Os consumidores brasileiros não dão a devida importância ao selo da fiscalização sanitária, sendo esta uma das últimas especificações verificadas e a data de validade a primeira especificação observada nas embalagens de alimentos (OLIVEIRA et al., 2010; RINALDI et al., 2010). No caso do leite caprino, grande parte dos con- 
sumidores adquire o leite e seus derivados direto dos criadores e não verifica se o produto é inspecionado (CELIA, 2010).

Embora estudos apontem que a fiscalização é um atributo observado pelos consumidores brasileiros na compra de carne (SPERS et al., 2003; ZAMUDIO, 2010), o consumo de carne não fiscalizada ainda é grande. No Rio Grande do Sul, estima-se que cerca de 6 mil ovinos são abatidos informalmente para consumo na própria propriedade rural ou em abates clandestinos, o que ocasiona prejuízos econômicos ao estado e pode acarretar graves problemas de saúde pública (AZAMBUJA e SANTOS, 2008). Na observação deste quesito para compra de alimentos, renda e escolaridade têm efeito significativo (ZAMUDIO, 2010).

No caso dos produtos coloniais, uma parcela dos consumidores não tem o hábito de comprar estes produtos por entenderem que não são seguros, indicando que a questão sanitária é um assunto importante na opinião dos consumidores (ARBAGE et al., 2007).

Outro sistema de certificação associado à qualidade do alimento é a rastreabilidade que passou a ser exigida como forma de garantir informação imediata e transparente sobre os perigos que possam afetar a segurança do alimento que consome. A rastreabilidade visa atender a essa necessidade, mantendo um registro confiável, ágil e seguro de todos os passos envolvidos nos processos da cadeia produtiva, desde o fornecedor de insumos até a disponibilização do produto ao consumidor (DIGIOVANI, 2006).

Como o termo rastreabilidade é recente e pouco divulgado, muitos consumidores possuem pouco conhecimento sobre o assunto e, em geral, associam-no somente à carne bovina. O consumidor brasileiro desconhece o significado da rastreabilidade e não tem confiança nas informações a respeito da origem e da qualidade da carne bovina que compra. Entretanto, quando informado sobre o significado deste processo de certificação, o consumidor passa a considerar a medida importante quanto à informação da origem e condições sanitárias do produto e a garantia de qualidade deste, considerando, inclusive, pagar um preço maior pelo produto rastreado (GONÇALVES et al., 2009 e BRISOLA et al., 2003).

No caso da carne de frango, rastreabilidade é um dos aspectos considerados menos importantes pelos consumidores do Distrito Federal (ZAMUDIO, 2010). Por outro lado, é um fator de decisão muito importante para os consumidores paulistas, quanto aos produtos orgânicos (LOMBARDI et al., 2007).

\section{CONCLUSÕES}

Entre os fatores que determinam o consumo de produtos certificados estão renda mensal, escolaridade e número de pessoas na família. Sendo o preço elevado um dos obstáculos ao consumo de produtos certificados. Um percentual significativo de consumidores ainda não sabe o que é certificação, o que se constitui em entrave para a decisão de compra dos produtos certificados. Estudos apontam incremento na importância que os consumidores atribuem à certificação após campanhas educativas. Neste sentido, faz-se necessário desenvolver campanhas de informação e de divulgação das características de produtos certificados. Estas campanhas constituem instrumentos básicos para o reconhecimento da necessidade e confiança no produto.

\section{REFERÊNCIAS}

ALCANTARA, N.B.; MOURAD, C.B.; CUNHA, C.F.; BIBANCOS, M. A opinião dos consumidores sobre os selos de qualidade de alimentos das redes de varejo. In: SEMEAD, 11,2008, São Paulo/ SP. Disponível em: <http://www.ead.fea.usp.br/ semead/11semead/resultado/an_resumo.asp?cod_ trabalho=637>. Acesso em: 27 fev. 2014.

ARBAGE, A.P.; SILVA, A.; SOUZA, R.S.; BAUMHARDT, E.; LISBOA, R.S. Comportamento de compra dos consumidores de produtos coloniais na região Central do Rio Grande do Sul. In: SOBER, 45, 2007, Londrina/PR.

AZAMBUJA, R.M.; SANTOS, D.V. Potencialidade de ovinos para abate no Rio Grande do Sul. Informativo Técnico DPA, v.1, n.1, p.3-5, 2010.

BARCELLOS, J.O.J.; ABICHT, A.MM.; BRANDÃO, F.S.; CANZZI, M.E.A.; COLLARES, F.C. Consumer perception of Brazilian traced beef. Revista Brasileira de Zootecnia. v.41, n.3, p.771-774, 2012.

BARROS, J.D.S.; FREITAS, L.S. Rotulagem ambiental: um estudo sobre os fatores de decisão de compra de produtos orgânicos. In: SIMPÓSIO DE EXCELÊNCIA EM GESTÃO DE TECNOLOGIA, 
7, 2010. Disponível em: <http://www.aedb.br/seget/ artigos10/459_Rotulagem $\% 20$ versao $\% 20$ final $\% 20$ com\%20autores.pdf>. Acesso em: 27 fev. 2014.

BRISOLA, M.V.; ESPÍRITO SANTO, E.; PALUDO, M.T.A. O interesse do consumidor da cidade de Brasília pela rastreabilidade da carne bovina. In: $\mathrm{CON}$ GRESSO INTERNACIONAL DE ECONOMIA E GESTÃO DE REDES AGROALIMENTARES, 4, 2003, Ribeirão Preto/SP.

CAETANO, M.R.; VALENTE, A.L.E.F.Construção social da qualidade: um estudo de caso sobre o consumo de queijos artesanais em Brasília. In: CONGRESO LATINOAMERICANO DE SOCIOLOGIA RURAL, 8, 2010. Porto de Galinhas, Pernambuco.

CERVO, A.L.; BERVIAN, P.A. Metodologia Científica.São Paulo: McGraw-Hill, 1983.

CHANG, H.S.; ZEPEDA, L. Consumer perceptions and demand for organic food in Australia: Focus group discussions. Renewable Agriculture and Food Systems. v.20, n.3, p.155-167, 2005.

DIGIOVANI, M.S. Certificação, rastreabilidade e normatização. Boletim Informativo daFAEP. n.705, p.8. 2006.

DRIES, L.; MANCINI, M.C. (Coord.).Food qualiry assurance and certification schemes. Bruxelas: European Community, 2006.

DUBOIS, B. Compreender o consumidor. Lisboa: Dom Quixote, 1993.

FREITAS, H.; OLIVEIRA, M.; SACCOL, A.Z.; MOSCAROLA, J. O método de pesquisa survey. Revista de Administração. v. 35, n. 3, p.105-112, 2000.

GIL, A.C. Métodos e técnicas de pesquisa social. 5. ed. São Paulo: Atlas, 2007.

GONÇALVES, A.A.; PASSOS, M.G.; BIEDRZYCKI, A. Percepção do consumidor com relação à embalagem de pescado: estudo de caso com os alunos do curso de engenharia de alimentos. Estudos Tecnológicos. v.5, n.1, p.14-32, 2009.

GRUNERT, K.G. Food quality and safety: consumer perception and demand. European Review of Agricultural Economics. v.32, n.3, p.369-391, 2005.
KIMURA, A.H. Between technocracy and democracy: An experimental approach to certification of food products by Japanese consumer cooperative women. Journal of Rural Studies. v.26, p.130-140, 2010 .

KUNESKI, M.; CAZELLA, A.A.; KARAM, K.F. Apoio a consumidores de produtos agroecológicos na região da grande Florianópolis. Revista Eletrônica de Extensão - UFSC. v.1, n.1,2004.

LOMBARDI, M.S.; MOORI, R.G.; SATO, G.S. Estudo exploratório dos fatores relevantes na decisão de compra de produtos orgânicos. Revista de Administração Mackenzie. v.5, n.1, p.13-34, 2004.

LOMBARDI, M.F.S.; PEROSA, B.B.; PEROSA, J.M.Y.; BUSO, D.R.; NASCIMENTO, F.M.A.Consumer Behavior for organic products in Botucatu - SP.Ribeirão Preto: USP, 2007.

OLIVEIRA, E.S.; RIBEIRO, M.B.; NOBRE, S.M.; SOUSA, F.R. Perfil, hábitos e atitudes do consumidor de carne bovina mirandesa. In: COLOQUIO IBÉRICO DE ESTUDIOS RURALES, 8, 2010.

RINALDI, R.N.; RAMOS, M.J.; FALCÃO,G.P. Percepções do consumidor relacionadas à qualidade esegurança dos alimentos - um estudo de caso aplicado nauniversidade aberta à terceira idade - UNATI DE TOLEDO/PR. In:CONGRESO LATINOAMERICANO DE SOCIOLOGIA RURAL, 8, 2010. Porto de Galinhas, Pernambuco.

RODRIGUES, R.M. Pesquisa Acadêmica: como facilitar o processo de preparação de suas etapas. São Paulo: Atlas, 2007.

ROMBALDI, C.V.; TIBOLA, C.S.; FACHINELLO, J.C.; SILVA, J.A. Percepção de consumidores do Rio Grande do Sul em relação a quesitos de qualidade em frutas. Revista Brasileira de Fruticultura. v.29, n.3, p.681-684, 2007.

SATO, G.S. As novas regras para o mercado global: certificações de origem e qualidade para alimentos seguros. Revista Eletrônica de Negócios Internacionais. v.4, n.1, p.151-163, 2009.

SPERS, E.E.; ZYLBERSZTAJN, D.; LAZZARINI, S.G. Percepção do Consumidor sobre os Mecanismos de Qualidade e Segurança em Alimentos. Revista de Administração da UNIMEP. v.1, n.1, p.57-80, 2003. 
THILMANY, D.D.; UMBERGER, W.J.; ZIEHL, A.R. Strategic market planning for value-added natural beef products: A cluster analysis of Colorado consumers. Renewable Agriculture and Food Systems. v.21, n.3, p.192-203, 2006.

THRUSFIELD, M. Epidemiologia Veterinária. 2. ed. São Paulo: Roca, 2004.

TIBOLA, C.S.; FACHINELLO, J.C. Tendências e estratégias de mercado para a fruticultura. Revista Brasileira de Agrociência. v.10, n.2, p.145-150, 2004.

VELHO, J.P.; BARCELLOS, J.O.J.; LENGLER, L.; AL-ALAM ELIAS, S.; OLIVEIRA, T.E. Disposição dos consumidores porto-alegrenses à compra de carne bovina com certificação. Revista Brasileira de Zootecnia. v.38, n.2, p.399-404, 2009.

VIVAS, C.I.R.; COELHO, M.D.C.M.; ESTEVES, M.I.C. Análise do comportamento de consumidor de queijos certificados alentejanos. In:AYALA CALVO, J.C.A. (coord). Conocimiento, innovación y emprendedores: Camino al futuro. Logroño/Espanha: Universidad de La Rioja, 2007. p. 2128-2141..

ZAMUDIO, L.H.B. Caracterização e percepção de consumidores sobre a qualidade da carne de frango comercializado em Brasília, DF. 2010. 125p. Dissertação (Mestrado em Agronegócios) - Universidade de Brasília, Brasília. 